\title{
Cutaneous Sinus Tract from Mandibular Second Molar with C-shaped Canal System and Improper Former Root Canal Treatment: A Case Report
}

\author{
Maryam Gharechahi ${ }^{1)}$ and Parisa Dastmalchi ${ }^{2)}$ \\ ${ }^{1)}$ Department of Endodontics, Dental Research Center, School of Dentistry, \\ Mashhad University of Medical Sciences, \\ P. O. Box: 91735-984, Mashhad, Iran \\ ${ }^{2)}$ School of Dentistry, Mashhad University of Medical Sciences, \\ P. O. Box: 91735-984, Mashhad, Iran
}

Received 3 December, 2015/Accepted for publication 28 January, 2016

\begin{abstract}
Here, we report the diagnosis and treatment of an extraoral cutaneous sinus tract originating in a mandibular second molar with a $\mathrm{C}$-shaped root canal system. The patient was referred to our department by a dermatologist after a series of unsuccessful treatments, including antibiotics. Diffuse radiolucency on a preoperative radiograph revealed that earlier root canal treatment had been only partially successful. Consequently, we performed retreatment of the root canal comprising removal of the former restoration and gutta-percha, cleaning and shaping, and passive irrigation with sodium hypochlorite. The patient responded well, and the cutaneous lesion completely resolved uneventfully within 1 month postoperatively. Preoperative recognition and thorough knowledge of the root canal anatomy and conventional methods of obturation are necessary in performing successful endodontic treatment.
\end{abstract}

Key words: C-shaped canal — Cutaneous - Fistula - Molar — Sinus tract

\section{Introduction}

Most cutaneous sinus tracts in the face and neck region are odontogenic in origin. When observed extraorally, however, they are often mistaken for a skin lesion, prompting the patient to seek treatment from a general physician, general surgeon, or dermatologist, who may also misdiagnose the problem ${ }^{1)}$. This may result in the patient having to undergo multiple surgical excisions, radiotherapy, biopsies, or antibiotic regimens unnecessarily. Moreover, as none of the treatments given would actually address the root cause of the problem, the danger of recurrence is high. On the other hand, surgical or non-surgical root canal treatment, root canal retreatment, and even tooth extraction have been shown to promote healing within a matter of weeks in such cases ${ }^{7}$. 

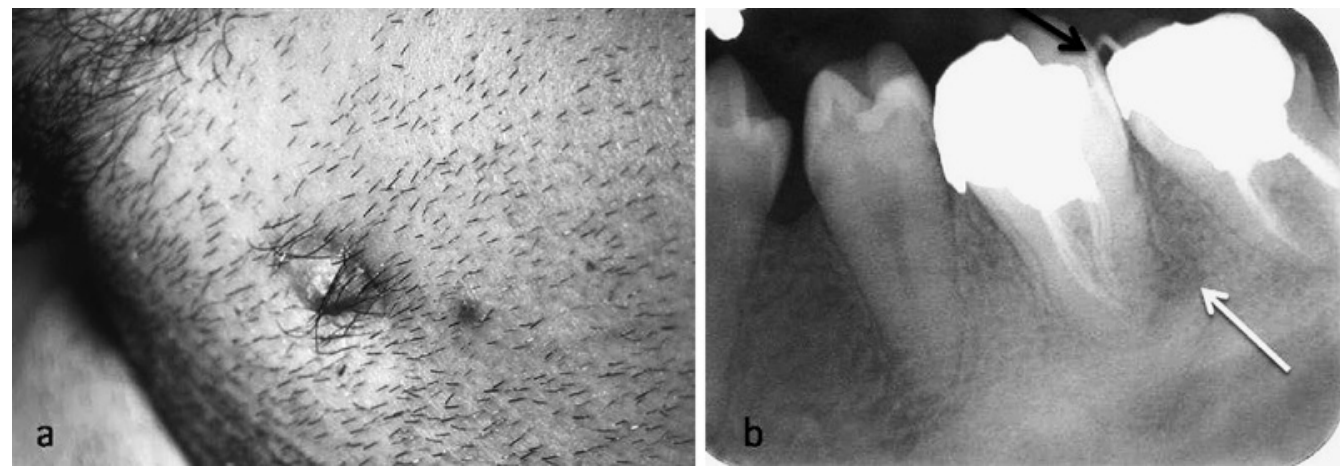

Fig. 1

a) Preoperative photograph

b) Preoperative diagnostic radiograph with tracing gutta-percha (black arrow). White arrow indicates periapical radiolucency

The posterior teeth, and especially the mandibular second molars, are characterized by considerable variation in root canal morphology ${ }^{4)}$, which makes them more likely to generate sinus tracts, potentially leading to serious consequences ${ }^{5,8)}$.

\section{Case Report}

A healthy 21-year-old man was referred to our department by his dermatologist with the chief complaint of a discharging sinus on the left cheek. The sinus had first appeared approximately 10 months earlier, since which time the patient had experienced repeated episodes of discharge. His medical history was noncontributory. The lesion comprised a painless indentation approximately $1.5 \mathrm{~cm}$ (0.6 in) in diameter on the left cheek containing a $4-\mathrm{mm}(0.15 \mathrm{in})$ friable papule with a yellow crust (Fig. 1a). The patient had already completed several courses of antibiotic therapy, all of which had proved ineffective. He had also been examined by several general physicians, an otolaryngologist, and a dermatologist before being referred to our department.

No vestibular swelling was observed intraorally, although the labial alveolar mucosa was slightly tender on palpation. The left man- dibular first molar (tooth \#36) had been extracted approximately 6 years earlier, and the mandibular second and third molars (teeth \#37 and \#38) had protracted into the space left by its removal. Tooth \#35 was distally inclined. Periapical radiography revealed that root canal treatment had been performed on teeth \#37 and \#38. It also showed a 1 -cm by 2 -cm ( 0.3 -in by 0.7 -in) radiolucency around tooth \#37, however. Although the root canal treatment on tooth \#38 was not ideal either, no periapical lesion was visible (Fig. 1b). Tooth \#37 was slightly tender on percussion, but tooth \#38 and the rest of the mandibular teeth responded within the normal limits. The teeth were firm (no detectable mobility) and no periodontal defects were evident on probing. Electric pulp and heat tests (using temporary stopping with guttapercha) were performed on all the left-side mandibular posterior teeth. Teeth \#37 and \#38 were nonresponsive to these stimuli, whereas the other teeth responded within the normal limits. A ProTaper gutta-percha cone (Dentsply Maillefer, Ballaigues, Switzerland) was inserted into the tract to trace its origin. The results showed that the tract led to the radiolucency surrounding the apex of tooth \#37 (Fig. 1b). Thus, it was confirmed that the initial endodontic treatment had only been partially successful, and that this had resulted 

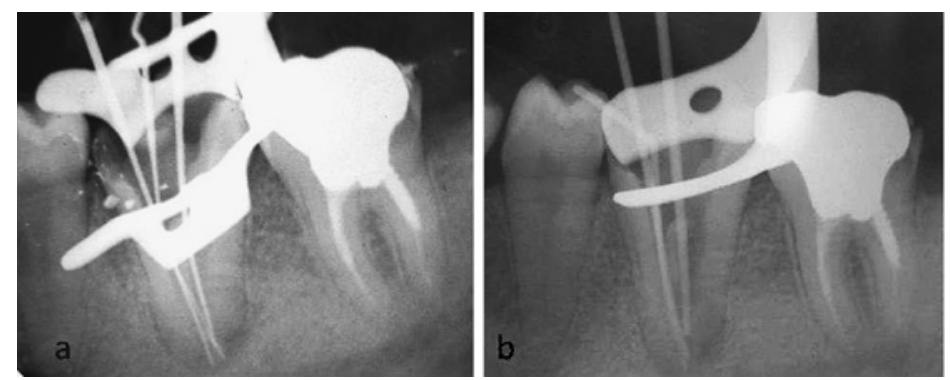

Fig. 2

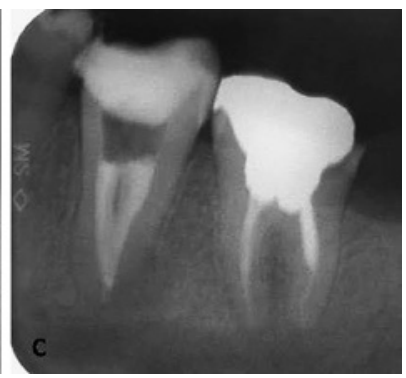

a) Working length radiograph with 3 file in place

b) Master point radiograph

c) Radiograph of obturated canal system

in the development of chronic suppurative periradicular periodontitis in that tooth. The treatment plan consisted of nonsurgical endodontic retreatment of tooth \#37 and root canal retreatment of tooth \#38 if necessary.

The presence of very close, and possibly fused, roots in the preoperative radiograph and the contours of the obturation suggested that the mandibular second molar had a C-shaped root canal system (Fig. 1b).

After adequate block anesthesia and isolation with a rubber dam, the previous restoration was removed. Observation of the floor of the pulp chamber, which was located deeply, revealed a type II (C2) C-shaped canal morphology (a separate mesiolingual canal and joined mesiobuccal and distal canals $)^{4}$. It is unsafe to use a Gates Glidden drill in a C-shaped canal as the head is inflexible and it is designed more for lateral cutting. Therefore, ultrasonic instruments, H-Files, and chloroform solvent were used instead to remove the old filling materials from the root canals. After complete removal of the guttapercha, the working length of the canals (WL) was determined using an electronic apex locator (Root ZX, J. Morita USA, Irvine, CA, USA) and confirmed radiographically (Fig. 2a). The radiograph also confirmed the joining of the three canals upon apical exit, which is common in C-shaped root canals ${ }^{8)}$. Cleaning and shaping in the separated root canal and wider portion of the $\mathrm{C}$-shaped canal was initiated with the Mtwo rotary system (Dentsply Maillefer), followed by circumferential instrumentation using hand instruments (Flexofile, Dentsply Maillefer). During preparation, $5.0 \mathrm{ml}$ of $5.25 \%$ sodium hypochlorite and $18 \%$ EDTA solution were employed for irrigation. This was followed by application of physiological saline and $1 \mathrm{~min}$ of passive ultrasonic irrigation with $4 \%$ sodium hypochlorite using the Piezon EMS (EMS GmbH, Munich, Germany) for maximum debridement of the complex anatomy of the $\operatorname{root}^{4}$.

After irrigation with saline, the root canals were dried with paper points (Dentsply Maillefer) and medicated with a calcium hydroxide-based paste (Quimis, Mallinkrodt, St. Louis, MO, USA) in a mixture with $2 \%$ chlorhexidine digluconate (Vista Dental Products, Racine, WI, USA) ${ }^{7}$. A 25-mm (1-in) \#4 Lentulo spiral RA (Dentsply Maillefer) was used to place the paste passively. The tooth was then closed with a sterile pledget cotton ball and temporary filling material (Cavit $\mathrm{G}^{\mathrm{TM}}$, 3M Espe, Seefeld, Germany).

The patient was recalled for follow-up treatment a week later. After isolation with a rubber dam and removal of the temporary restoration and calcium hydroxide paste, the canals were again thoroughly cleaned, but again not yet shaped because of the danger of perforating the narrow isthmus. The canals were irrigated again with $17 \%$ EDTA and 


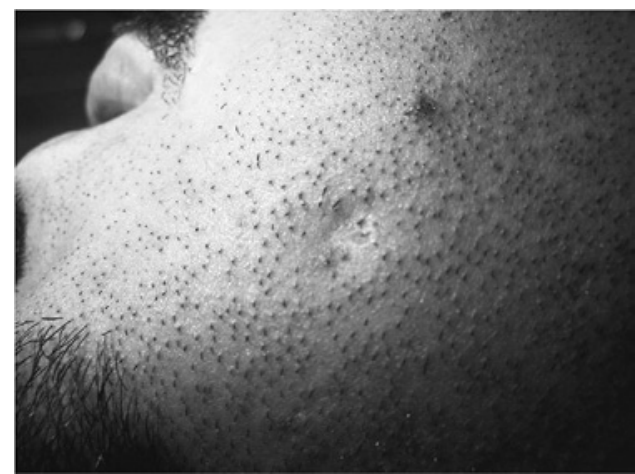

Fig. 3 1-month postoperative photograph showing healed sinus tract area on left cheek

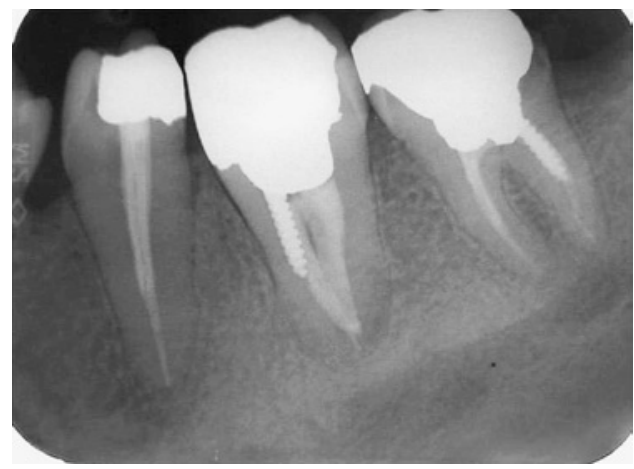

Fig. 4 2-year postoperative radiograph

\section{Discussion}

$5.25 \%$ sodium hypochlorite and dried with paper points. The master point was seated and confirmed radiographically (Fig. 2b). Finally, the tooth was obturated by the thermoplasticized gutta-percha method using Obtura II (Obtura Spartan, Fenton, MO, USA) with ProTaper gutta-percha (Dentsply Maillefer) and AH Plus root canal sealer (Dentsply DeTrey GmbH, Konstanz, Germany) (Fig. 2c).

After sealing the tooth with Cavit, the patient was referred to the Restoration Department for a permanent amalgam restoration, with instructions that the mesial canals should be used if a post was required because of the unusual C-shaped configuration of the distal canal. It was also advised that retreatment of the mandibular third molar be reconsidered.

The first follow-up appointment was scheduled for 1 week later, although the patient was also asked to return earlier if he experienced any persistent or progressive pain or any other abnormality before then or any other scheduled visit. The cutaneous lesion healed and the friable papule resolved within 1 month of treatment (Fig. 3); no evidence of abscess recurrence was observed throughout 2 years of follow-up visits (Fig. 4).
The reason infections sometimes spread via an extraoral pathway is unknown. Some researchers have suggested that there is a higher chance that an infection will do so if the apex of the tooth is situated superior to the muscle attachments ${ }^{5}$. Other major risk factors that increase the chance of external sinus tract occurrence are bacterial virulence and body resistance ${ }^{6)}$.

Oral sinus tracts of odontogenic origin are often initially misdiagnosed, resulting in treatment that is inappropriate. Therefore, the evaluation of a cutaneous sinus tract must involve a thorough check of the patient's medical history together with a dental examination to investigate possible symptoms of dental caries, oral trauma, periodontal disease, or poor oral hygiene. A dental examination is crucial here, as a cutaneous tract can easily be mistaken for a cyst, furuncle, or ulcer $^{1)}$.

The differential diagnosis of a cutaneous draining sinus tract should include suppurative apical periodontitis, osteomyelitis, congenital fistula, salivary gland fistula, infected cyst, and deep mycotic infection ${ }^{1)}$. Skin lesions, such as pustules, furuncles, foreignbody lesions, squamous cell carcinoma, and granulomatous disorders may be superficially similar in appearance to a draining sinus tract of odontogenic origin, but they are not true sinus tracts $^{7}$. It should be pointed out that 
since the sinus tract provides drainage, it prevents swelling or pain. As a result, the presence of acute pain, cutaneous lesions or swelling is seldom related to sinus tracts of odontogenic origin ${ }^{2}$.

Here, we described a case in which initial endodontic treatment had been unsuccessful and in which an unusual anatomy, a C-shaped root canal system, was present. There are many reasons $\mathrm{C}$-shaped canals can present a challenge in reaching a diagnosis and successfully achieving debridement and obturation, among which morphological variation along the length of the roots, particularly the apical third, is the most important ${ }^{4)}$. The isthmus between the canals is not thick as such canals are not geometrically in the center of the roots and there is a higher risk of striping and perforation $^{3)}$. Over-preparing the orifice might lead to perforation into the lingual groove because of the deep pulp chamber. Furthermore, the presence of a high incidence of transverse anastomoses, lateral canals, and apical deltas makes cleaning and sealing the root canal system much more difficult ${ }^{5)}$. All these factors render the treatment of Cshaped root canal systems more difficult.

In the present patient, the partial failure of earlier root canal treatment was identified as the main reason behind the formation of the sinus tract. The presence of a C-shaped root canal system was detected and retreatment performed. The cutaneous lesion healed within 1 month of treatment, and no evidence of abscess recurrence was observed during the 2 years of subsequent follow-up visits.

\section{Conclusion}

The early and accurate diagnosis of an extraoral sinus tract can prevent unnecessary and ineffective rounds of antibiotic therapy and surgical intervention.

\section{Acknowledgement}

The authors would like to thank the Vice-
Chancellor of Research at Mashhad University of Medical Sciences for financial support for this research.

\section{Conflict of Interest}

The authors declare no conflict of interest related to this study.

\section{References}

1) Bai J, Ji AP, Huang MW (2014) Submental cutaneous sinus tract of mandibular second molar origin. Int Endod J 47:1185-1191.

2) Cantatore JL, Klein PA, Lieblich LM (2002) Cutaneous dental sinus tract, a common misdiagnosis: a case report and review of the literature. Cutis 70:264-267.

3) Helvacioglu-Yigit D (2015) Endodontic management of C-shaped root canal system of mandibular first molar by using a modified technique of self-adjusting file system. J Contemp Dent Pract 16:77-80.

4) Jafarzadeh H, Wu YN (2007) The C-shaped root canal configuration: a review. J Endod 33: 517-523.

5) Kato A, Ziegler A, Higuchi N, Nakata K, Nakamura H, Ohno N (2014) Aetiology, incidence and morphology of the C-shaped root canal system and its impact on clinical endodontics. Int Endod J 47:1012-1033.

6) Sammut S, Malden N, Lopes V (2013) Facial cutaneous sinuses of dental origin-a diagnostic challenge. Br Dent J 215:555-558.

7) Wigler R, Steinbock N, Berg T (2013) Oral cutaneous sinus tract, vertical root fracture, and bisphosphonate-related osteonecrosis: a case report. J Endod 39:1088-1090.

8) Woelber JP, Bruder M, Tennert C, Wrbas KT (2014) Assessment of endodontic treatment of c-shaped root canals. Swiss Dent J 124: 11-15.

Correspondence:

Dr. Maryam Gharechahi

Department of Endodontics, Faculty of Dentistry,

Mashhad University of Medical Sciences, P. O. Box: 91735-984, Mashhad, Iran E-mail: gharechahim@mums.ac.ir 